\title{
Counseling About Traction Alopecia: A "Compliment, Discuss, and Suggest" Method
}

\author{
Ciara Grayson, MD; Candrice R. Heath, MD
}

\section{PRACTICE POINTS}

- When communicating with patients regarding traction alopecia (TA), it is crucial to display cultural humility and empathy.

- Understanding the patient's hair care goals and perspective allows dermatologists to take a more individualized approach to counseling about TA.

- The "compliment, discuss, and suggest" method is an empathetic and culturally sensitive method for discussing TA with patients.

Traction alopecia (TA) remains a common concern for Black women visiting a dermatologist. Understanding cultural hair care practices, timely diagnosis, and discontinuation of high-risk hairstyles are essential for preventing TA from progressing to scarring alopecia. This dialogue might be difficult for hair-discordant physicians and patients because of a lack of training and shared experiences. We present an empathetic and culturally sensitive method for discussing TA with patients.

Cutis. 2021:108:20-22.

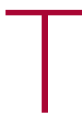

raction alopecia (TA) - one of the most common types of hair loss in Black women (although not exclusive to Black women)-is reversible when early corrective measures are taken; if chronic tension continues, however, permanent scarring alopecia ensues. Dermatologists can prevent worsening of this distressing hair loss. Due to a dearth of training among dermatologists in conditions occurring in patients with tightly coiled hair, it is imperative to add practical methods to the body of dermatology literature, with the goal of enhancing cultural humility.

Hairstyling among Black women often is a lengthy process and often results in relationship bonding with the hair care giver, in turn imparting hair care traditions to the next generation. Therefore, a well-received discussion about TA prevention not only has an impact on the patient but potentially on a multigenerational family of women and friends. We present a memory aid for discussing TA, with a focus on cultural humility and patientcentered communication.

Factors contributing to the risk of TA are hairstyles and hair care practices commonly used in Black individuals, including braids, locs, weaves, wigs, and chemical straightening. ${ }^{1}$ These styles often are worn to increase hair manageability or as a creative expression of beauty.

Discussing TA can be distressing for physicians and patients, especially in the setting of hair texture discordance. In a study that surveyed Black patients' perception of their dermatologic care both in and outside of a skin of color clinic, $71 \%$ of respondents (12/17) said that they prefer a race-concordant dermatologist. Some

Dr. Grayson is from the Florida State University College of Medicine Internal Medicine Residency Program, Tallahassee. Dr. Heath is from the Department of Dermatology, Lewis Katz School of Medicine, Temple University, Philadelphia, Pennsylvania. 
respondents reported that non-skin of color clinic dermatologists examined their hair with the end of a pencil or not at all; patients interpreted these interactions as disrespectful and racially insensitive. ${ }^{2}$ Another study found that only $30.2 \%(19 / 63)$ of dermatology chief residents and $12.2 \%(5 / 41)$ of program directors reported a specific rotation during which residents gained experience treating skin of color patients. ${ }^{3}$

Due to a paucity of training in diagnosing and treating patients with tightly coiled hair who experience hair loss, some physicians might feel uncomfortable caring for patients who have tightly coiled hair. Although many Black patients prefer to see a race-concordant dermatologist because of their perceived cultural competence and shared experience, there is a paucity of Black dermatologists to see all patients who have tightly coiled hair. ${ }^{4}$ Therefore, all dermatologists should become skilled and comfortable discussing and treating TA in patients with all hair types.

\section{METHOD FOR COUNSELING}

The following scenarios are a guide to begin closing the competency gap in counseling about TA, using a "compliment, discuss, and suggest" method.

\section{Scenario 1}

A Black woman presents with a concern of "thinning edges" (a popular term on social media for TA). A hairdiscordant dermatologist tells her, first, that she has TA caused by wearing tight hairstyles and, second, that the treatment is to stop wearing tight braids and weaves and to discontinue chemical relaxers. The dermatologist then leaves the room.

The Patient's Perspective - It is not uncommon for the patient to have feelings of frustration about how they will style their hair, especially if they are unfamiliar with caring for their hair in its natural state. ${ }^{5}$ Also, they might have feelings of dismay that the loving childhood hair care giver, often their mother or grandmother, unintentionally harmed them with a tight style. They also might feel betrayed by their hairstylist, who might not have encouraged them to see a dermatologist, or who continued to oblige their request for a high-risk hairstyle. The patient might feel uncomfortable communicating the dermatologist's new recommendations to their hair care team, who also are part of her emotional support system. The patient also might think that the hair-discordant dermatologist has no idea what they "go through" with their hair.

"Compliment, Discuss, and Suggest" CounselingTraction alopecia is caused by tight hairstyles that often hurt when they are put in as tight braids, weaves, and ponytails. ${ }^{6}$ Risk increases if tight styles are applied to chemically straightened hair. ${ }^{1}$ Braids, sew-in weaves, and wigs with adhesive sometimes are referred to as protective styles. However, these styles can still lead to TA due to excessive tension.
- Compliment: "Your hair looks great. I know that you get many compliments."

- Discuss: "However, some of the styles might be increasing your risk for hair loss. Our goal is to preserve as many of your follicles as possible."

- Suggest: "Let's start by loosening the hairstyle if it is painful when being applied. Pain means inflammation, which can lead to scarring of hair follicles and worsening of hair loss."

Using pronouns such as we, us, and our is intentional. Doing so signals that the dermatologist is a partner with the patient in the treatment of TA. Starting with a simple initial recommendation gives the patient time to process the common thoughts highlighted in The Patient's Perspective section. ${ }^{6}$

\section{Scenario 2}

A Black child (we'll call her "Janet") is accompanied by her mother for follow-up of mild atopic dermatitis on the body and scalp. When the dermatologist examines the patient's scalp, they note that she has the fringe signretained short hairs along the frontal hairline-that is consistent with TA. Janet's hair is adorned with 2 tight ponytails in the front with colorful decorative balls on ponytail ties, barrettes, and 6 cornrow braids in the back with plastic beads on the ends. The dermatologist counsels about the atopic dermatitis and leaves the room.

"Compliment, Discuss, and Suggest" Counseling-The use of tight decorative balls on ponytail ties and numerous plastic beads increases the amount of tension and weight on the hair, which may lead to a higher risk for developing traction alopecia. ${ }^{6}$ It is quite common for children of African descent to wear hair adornments. Proper counseling regarding their use and possible implications is essential.

- Compliment: “You're doing a great job controlling the atopic dermatitis, which can cause Janet's scalp to be dry. Also, her hair is beautiful-it looks like you spent a lot of time on her hair. And Janet, I like the color of your barrettes."

- Discuss: "Mom, I just noticed that a few areas look tight. Let's look together." (The dermatologist points out areas where the scalp is tented upward due to traction, follicular pustules or papules, or the frontal fringe sign.) "I'm on a mission to \#savetheedges because we want Janet to grow up with full edges." (Again, loss of "edges" refers to TA.)

- Suggest: “When you do Janet's hair, it's OK if every hair is not in place. In fact, making styles look and feel 1 or 2 weeks old will lessen tension on the scalp. Remove Janet's hair ties to release tension when she is at home and while she's sleeping, if possible. Every minute that the hair is loose really does help."

The Parent's Perspective-All parents take pride in their children. In some Black communities, mothers are judged by how well they manage and style their children's hair. Some people might even suggest that parents of children 
with nonstyled, tightly coiled hair are not fit parents. Anthropologist Sylvia Boone, $\mathrm{PhD}$, found that among the Mende tribe in Sierra Leone, "unkempt, 'neglected,' or 'messy' hair implied that a woman either had loose morals or was insane." ${ }^{7}$

Braids are commonly worn by people of African heritage for a variety of reasons, including ease of manageability, to decrease daily hairstyling time, and as an expression of creativity. Intricate neat hairstyles, despite the risk of pain and TA, are perceived as a sign that the child is cared for and loved. ${ }^{6}$

\section{FINAL THOUGHTS}

Patient-centered communication is associated with the patient trusting the physician, which is especially important in race-discordant physician-patient relationships. A study found that patient-physician race discordance led to shorter visits, a lower rating of patient affect, and less shared decision-making. ${ }^{8}$ Moreover, in a study of primary care clinicians, implicit bias was found to affect communication patterns and social interactions, impacting patient outcomes. Downstream effects of racial bias resulted in less speaking, smiling, and social comments when interacting with Black patients. ${ }^{9}$

These findings highlight the need to address interpersonal barriers to effective communication in racediscordant patient-physician dyads. A history of segregated neighborhoods and schools might contribute to structural barriers, resulting in lack of familiarity with cultural norms outside one's culture, which might globally perpetuate poor communication and patient outcomes.

The "compliment, discuss, and suggest" method might lead to more positive physician-patient encounters by having the dermatologist focus on empathetically understanding the patient's perspective. ${ }^{10}$ Effective communication, understanding cultural hair care practices, and a thorough scalp examination are paramount for patients with tightly coiled hair. ${ }^{11}$ Early intervention in TA is crucial and involves partnering with patients and parents to amend high-risk hairstyling routines with cultural humility.

\section{REFERENCES}

1. Haskin A, Aguh C. All hairstyles are not created equal: what the dermatologist needs to know about black hairstyling practices and the risk of traction alopecia (TA). J Am Acad Dermatol. 2016;75:606-611. doi:10.1016/j.jaad.2016.02.1162

2. Gorbatenko-Roth K, Prose N, Kundu RV, et al. Assessment of Black patients' perception of their dermatology care. JAMA Dermatol. 2019;155:1129-1134. doi:10.1001/jamadermatol.2019.2063

3. Nijhawan RI, Jacob SE, Woolery-Lloyd H. Skin of color education in dermatology residency programs: does residency training reflect the changing demographics of the United States? J Am Acad Dermatol. 2008;59:615-618. doi:10.1016/j.jaad.2008.06.024

4. Lester J, Shinkai K. Diversity and inclusivity are essential to the future of dermatology. Cutis. 2019;104:99-100.

5. Gathers RC, Mahan MG. African American women, hair care, and health barriers. J Clin Aesthet Dermatol. 2014;7:26-29.

6. Heath C. Clinical pearls for kids, tweens, and teens with skin of color. Presented at: Skin of Color Update 2020; September 12-13, 2020.

7. Byrd AD, Tharps LL. Hair Story: Untangling the Roots of Black Hair in America. St. Martin's Griffin; 2002.

8. Cooper LA, Roter DL, Johnson RL, et al. Patient-centered communication, ratings of care, and concordance of patient and physician race. Ann Intern Med. 2003;139:907-915. doi:10.7326/0003-4819-139-11 -200312020-00009

9. Cooper LA, Roter DL, Carson KA, et al. The associations of clinicians' implicit attitudes about race with medical visit communication and patient ratings of interpersonal care. Am J Public Health. 2012;102:979-987. doi:10.2105/AJPH.2011.300558

10. Hashim MJ. Patient-centered communication: basic skills. Am Fam Physician. 2017;95:29-34.

11. Grayson C, Heath C. An approach to examining tightly coiled hair among patients with hair loss in race-discordant patientphysician interactions. JAMA Dermatol. 2021;157:505-506. doi:10.1001 /jamadermatol.2021.0338 